\title{
A New Method for the Prediction of Laser Cut Surface Topography
}

\author{
M. Harničárová ${ }^{1}$, J. Valíček ${ }^{2,3}$, M. Kušnerová ${ }^{2,3}$, R. Grznárik ${ }^{1}$, J. Petrů ${ }^{4}$, L. Čepová $^{4}$ \\ ${ }^{1}$ Nanotechnology Centre, VŠB - Technical University of Ostrava, 17. listopadu 15/2172, 70833 Ostrava-Poruba, \\ Czech Republic, corresponding author: marta.harnicarova@vsb.cz \\ ${ }^{2}$ Institute of Physics, Faculty of Mining and Geology, VŠB - Technical University of Ostrava, 17. listopadu 15/2172, \\ 70833 Ostrava-Poruba, Czech Republic \\ ${ }^{3}$ RMTVC, Faculty of Metallurgy and Materials Engineering, VŠB - Technical University of Ostrava, 17. listopadu 15/2172, \\ 70833 Ostrava-Poruba, Czech Republic \\ ${ }^{4}$ Department of Working and Assembly, Faculty of Mechanical Engineering, VŠB - Technical University of Ostrava, \\ 17. listopadu 15/2172, 70833 Ostrava-Poruba, Czech Republic
}

\begin{abstract}
The submitted contribution focuses on the clarification of a laser beam cutting technology especially from the point of view of created surface topography. It provides a new view on a deformation process caused by laser beam action and on possibilities of using the surface topography. The measurement and characterisation of surface topography was performed in depth traces using a contact profilometer Surftest SJ 401 and by and an optical-contact profilometer Talysurf CLI 2000 (measured from the top edge of the sample). Thanks to this procedure, it was possible to observe and to measure a development of the numerical values of the surface (profile) roughness parameter $R a$. Based on the measurement of the surface topography, there were analyzed and interpreted data with a purpose to theoretically describe surface topography and to develop an analytical solution for the profile topographical function. By using the profile topographical function, it is possible to solve the practical problems the most engineers and users face in laser beam cutting technology (LBC) process, as well as to maximize LBC manufacturing system performance and to determine the values of the process parameters that will reach the desired product quality.
\end{abstract}

Keywords: Laser cutting, surface topography, prediction, deformation parameters, topographic function

\section{INTRODUCTION}

$\mathrm{T}$ HE LASER CUTTING TECHNOLOGY is still described as a non-traditional machining method. In non-traditional machining processes a material is removed as a result of very complicated physical, electrochemical and mechanical phenomena. In LBC (laser beam cutting) the material is removed as a result of thermal processes. The laser beam is emitted by a laser focused on a very small surface of the machined material, which causes the power density of the laser beam to be very high (108 $1014 \mathrm{~W} \cdot \mathrm{cm}^{-2}$ ). Properties of the surface layer generated in the LBC process depend mainly on the power of the laser beam and power density on the machined surface. Currently, the LBC technology is still subject of concentrated research, as it is evident by a number of various publications. Many of experimental studies were designed to understand the factors affecting the performance of the cutting process [1], [2], [3]. The laser cutting technology and the accompanying phenomena occurring in the process of laser beam cutting or laser beam welding have been and still are the subject of many research activities with a view to optimizing technology parameters influencing cut or weld quality [4], [5], [6], [7]. The quality does not depend only on the choice of a suitable laser source for cutting a specific material, but considerable attention should be paid above all to process parameters. The optimum choice of process technology factors based on theoretical assumptions is connected with achieving a high-quality product and process [7], [8], [9]. The majority of theoretical studies are oriented to the presentation of authors' own results but without deep analyses. On the basis of analysis of available sources in the area of laser cutting, it can be critically stated that small attention is paid to the topographic function in the case of laser cutting and that any model of this function has not been systematically and comprehensively dealt with and published yet. All over the world, this issue is considered to be theoretically unsolved, without theoretical results applied in practice and without a possibility of exact approach to the design and proposal for the optimum parameters of material laser cutting [7], [8], [9]. These authors confirm that the mechanism of laser cutting as a mechanically flexible tool with thermal effects and many other factors participating in the mechanism of material disintegration is, from the point of view of analytical approximation, elaboration and description, very problematic. The knowledge of the topographic function is of high importance to other analyses and prediction of condition of surface topography at a change in technology regime as well as at the feedback control of final surface roughness [4], [5], [6], [10].

The analytical knowledge of regularities of the zonal distribution of values of geometric and stress-deformation parameters is a precondition for developing the prediction equations that are, in consequence, of importance not only to the choice of a suitable technology but also to the automation of machining process. The insufficient level of existing knowledge in the field of laser cutting can be formulated into the following parameters:

- insufficient respecting the physics-mechanical parameters of materials, including the absence of their uniform mathematical formulation,

- insufficient respecting the physics-mechanical parameters, and stress and deformation integrity of the 
system: technology parameters-tool-material-surface topography,

- insufficient utilizing the regularities of distribution and zonality of geometric parameters of cut wall surface produced by the laser beam,

- inconsistency in the interpretation of results achieved,

- absence of a theoretical prediction of limits of depths achievable in various materials.

It is very important to get a deep knowledge of the laser cutting process in relation to theory and industrial practice. During the thermal machining of materials, complicated dynamic phenomena appear. It is necessary to consider them also in the theory of machining in connection with the properties of this technology system: machine - workpiece tool, with relations between these properties and regularities of formation of new surfaces, and the related topographic function and generated deformation [11].

\section{THEORETICAL BACKGROUND}

The basic difference between the existing approach to the theoretical explanation of topographic function and the proposed way consists in the fact that the majority of research works regard the material as being constant in the course of machining. But the reality is that the physicomechanical properties of the material being machined change in the cutting process. During the cutting process, original physico-mechanical properties are influenced by generated stress and deformation and the temperature itself. In contrast to the hitherto approach, we choose our own path and put emphasis on the mechanical and stress-deformation parameters of the material being cut and on the mechanical equilibrium of the system: material properties-tool properties-deformation properties. The chosen procedure is then a solution to an implicit equation $R a=f\left(h, R e, W_{\text {las }}\right.$, $\left.I N D_{v p w}, Y_{\text {ret }}, \delta, v_{p}, p, d,(+/-) f, d T / d t, d T / d h, T_{\text {melt }}\right)$, where $h$ is the depth of cut, $R e$ is the yield strength, $W_{\text {las }}$ is the laser power, $I N D_{v p w}$ is the ratio (index) between traverse speed and laser power, $Y_{\text {ret }}$ is the retardation of the cut trace, $\delta$ is the deviation angle of trace, $v_{p}$ is the traverse speed, $p$ is the gas pressure, $d$ is the nozzle diameter, $(+/-) f$ the focal length, $d T / d t$ is the rate of temperature change in a time interval, $d T / d h$ is the rate of temperature change in the depth interval and $T_{\text {melt }}$ is the melting point. The quality of machining is characterised by the degree of precision of shape, dimensions and condition of surface layer after machining and related surface roughness [10]. It is thus advisable to direct attention to the topographic function, which will deepen the knowledge and the concept of basic research when investigating derived questions, such as material machinability or machining process rationalization. Researches as a result of which a uniform method of material machinability assessment, based on the physical principles of cutting process, would be developed are still topical. The aim of performed experiments in the interpretation and analysis of results is to determine a joint connection between the setting of input parameters of the laser cutting process and the final topography of machined surface of the sample. On the basis of results of surface topography measurement, the cutting process can be optimized to make the production process controlling surface topography quality more efficient.

\section{EXPERIMENTS}

In order to identify surface roughness, samples of $150 \times$ $150 \mathrm{~mm}$ size of different thicknesses were cut and different types of material were used. The cutting process was carried out using the optimum machine settings, i.e. the settings proposed by a control system after entering the parameters of the material being cut. For the realization of experiments a cutting machine from Prima Industry - ZAPHIRO / CV5000 (Fig.1), i.e. a 2D laser cutting system was used. The selected laser source was a $\mathrm{CO}_{2}$ laser with a wavelength of $10.6 \mu \mathrm{m}$. Parameters for cutting the used materials are given in Table1 and mechanical parameters of the used materials are given in Table 2, 3, 4 .

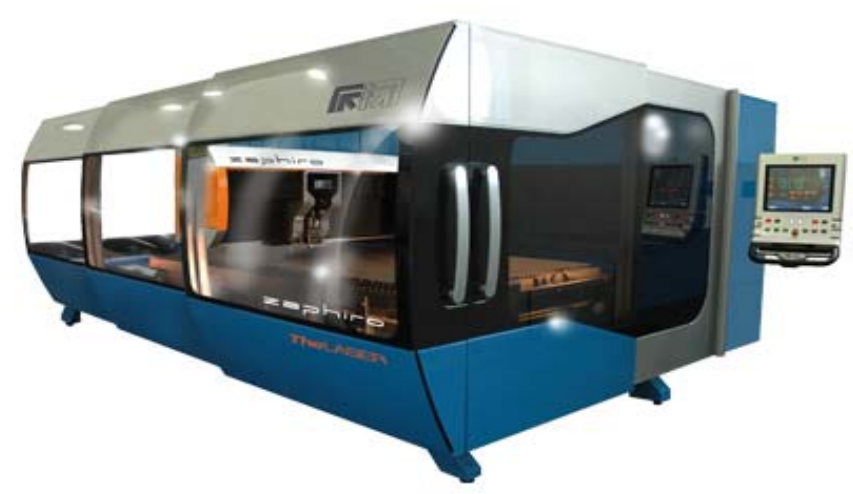

Fig.1. CNC 2D / 3D laser Prima Industrie, type Zaphiro/CV5000.

Table 1. Used parameters for cutting.

\begin{tabular}{|l|c|c|c|}
\hline \multirow{2}{*}{ parameters } & \multicolumn{3}{|c|}{ material } \\
\cline { 2 - 4 } & steel & stain. steel & titanium \\
\hline thickness $h[\mathrm{~mm}]$ & 20 & 15 & 10 \\
\hline lens $[$ inch] & $9^{\prime \prime}$ & $7.5^{\prime \prime}$ & $7.5^{\prime \prime}$ \\
\hline speed $v_{p}\left[\mathrm{~mm} \cdot \mathrm{min}^{-1}\right]$ & 830 & 1200 & $350,450,550$ \\
\hline power $P[\mathrm{~W}]$ & 4600 & 5000 & 3500 \\
\hline assist gas & $\mathrm{O}_{2}$ & $\mathrm{~N}_{2}$ & $\mathrm{~N}_{2}$ \\
\hline pressure $p[\mathrm{bar}]$ & 0.6 & 15 & 17 \\
\hline stand-off distance $z[\mathrm{~mm}]$ & 1.2 & 0.8 & 1.5 \\
\hline focus $[\mathrm{mm}]$ & 2 & -3 & $+/-$ \\
& & & $3.5 ;+2 ;+3$ \\
\hline nozzle diameter $d[\mathrm{~mm}]$ & 2.5 & 2 & 2 \\
\hline
\end{tabular}

Table 2. Mechanical parameters of EN S355J0.

\begin{tabular}{|c|c|c|c|}
\hline$R_{e}[\mathrm{MPa}]$ & $R_{m}[\mathrm{MPa}]$ & $A[\%]$ & $E_{\text {mat }}[\mathrm{GPa}]$ \\
\hline 360 & $510-628$ & 23 & 206 \\
\hline
\end{tabular}

Table 3. Mechanical parameters of X5CrNi18-10.

\begin{tabular}{|c|c|c|c|}
\hline$R_{e}[\mathrm{MPa}]$ & $R_{m}[\mathrm{MPa}]$ & $A[\%]$ & $E_{\text {mat }}[\mathrm{GPa}]$ \\
\hline 195 & $490-686$ & 45 & 200 \\
\hline
\end{tabular}

Table 4. Mechanical parameters of titanium Grade 2.

\begin{tabular}{|c|c|c|c|}
\hline$R_{e}[\mathrm{MPa}]$ & $R_{m}[\mathrm{MPa}]$ & $A[\%]$ & $E_{\text {mat }}[\mathrm{GPa}]$ \\
\hline $172-310$ & 345 & $25-37$ & 103 \\
\hline
\end{tabular}


The machined surfaces were subsequently measured using a contactless profilometer 2D Surftest SJ 401 and an opticalcontact profilometer Talysurf CLI 2000 (Fig.2). This system used for active measuring the surface roughness and form should have a low sensitivity to vibration, dustiness and other factors that impede measurement [12]. A solution to the topographic function is based on the physical-material principle and mathematical and mechanical aspects of the analysis of stress and deformation of the material being cut.

Study of literature, experiments and finally analytical deduction of conclusions are required separately for each specific condition of the technology process. From this point of view, it is needed to identify a whole set of technical, technological and material factors that enter into the cutting process. In the modern mathematical physics literature the standard way to reason about the measurement of a classical physical quantity or more generally about any empirical measurand (e.g., mass, electric charge, potential energy, pressure, stress, viscosity, temperature, humidity, utility, fitness, and so on) is in terms of a real-valued function that can in principle be measured or evaluated by a physically possible measuring instrument [13].

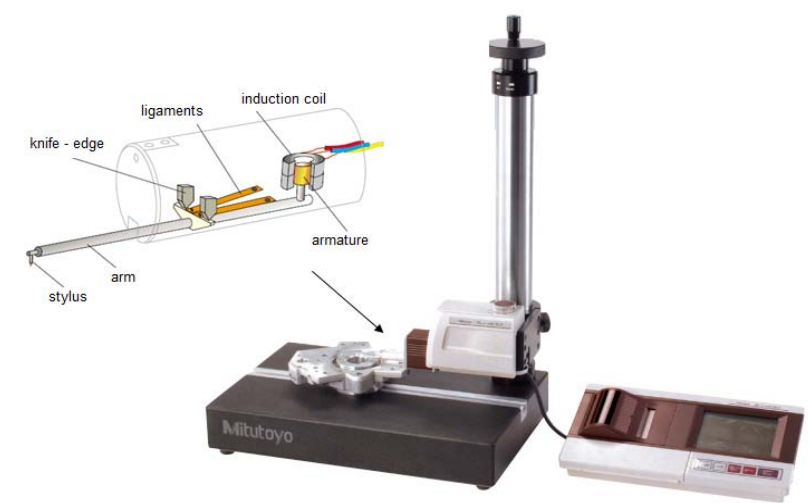

a)
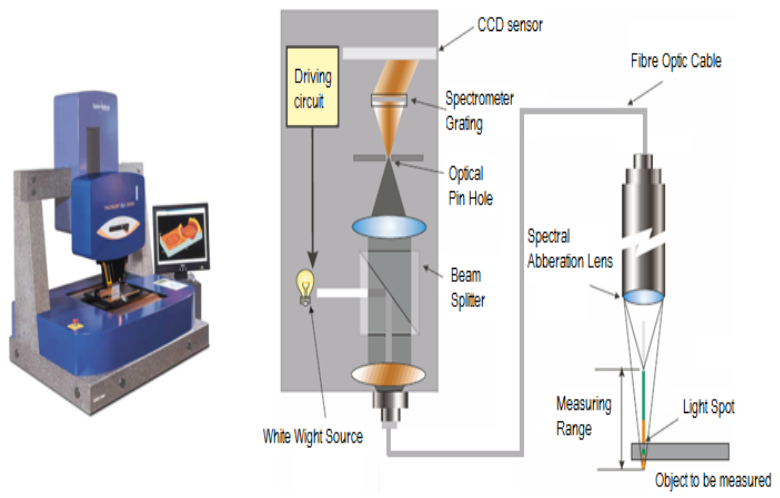

b)

Fig.2. Instruments used for measurement: a) Surftest SJ 401 produced by Mitutoyo, b) Talysurf CLI 2000 produced by TaylorHobson.

Surface topography profile obtained by Talysurf CLI 2000 is shown in Fig.3a and by the Surftest is shown in Fig.3b.

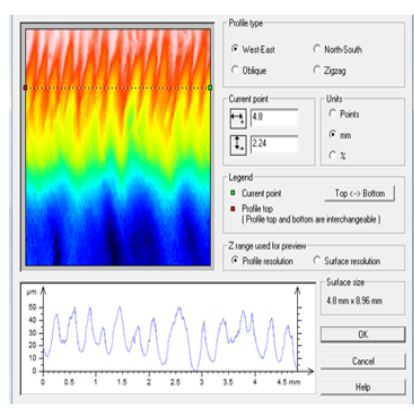

a)

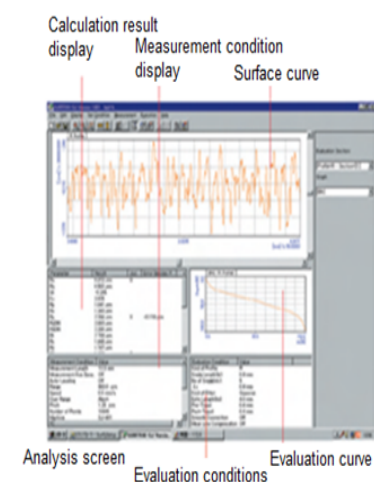

b)
Fig.3. Surface profile obtained by using of: a) Talysurf CLI 2000 b) Surftest SJ 401 .

\section{ANALYSIS AND INTERPRETATION}

The mechanism of cutting by means of a laser beam as a mechanically flexible cutting tool with thermal effects and many other factors entering into the mechanism of material disintegration is very problematic [1], [2], [3]. Thanks to experimentally performed measurements a development of numerical values of the surface (profile) roughness parameter $R a$ could be observed and measured. Based on these measurements, data were analysed and interpreted for the purpose of theoretical description of surface topography. The principle of the subject matter of our solution consists in the selection of a depth $h_{x}[\mathrm{~mm}]$ on the check cut wall with well visible traces after the cutting tool, where local roughness $R a_{x}[\mu \mathrm{m}]$ and retardation of the cut trace from the normal plane $Y_{\text {retx }}[\mathrm{mm}]$ are measured (Fig.4).

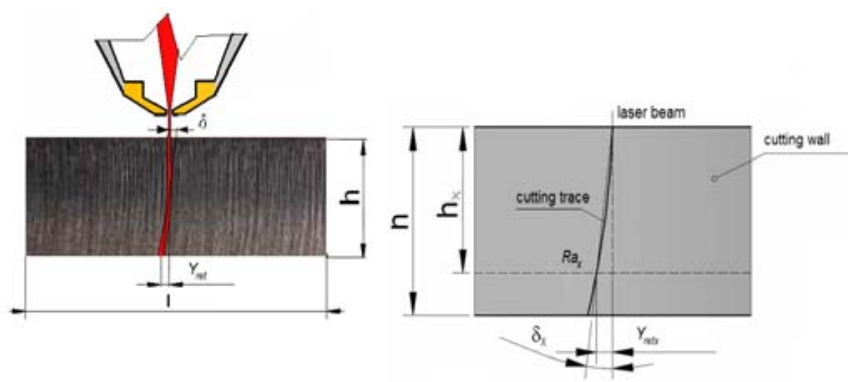

Fig.4. Determination of deformation parameters $R a_{x}, Y_{\text {retx }}, \delta_{x}, h_{x}$ at selected point $\mathrm{X}$ on cut wall after $\mathrm{CO}_{2}$ laser cutting.

In our analysis we proceed with an assumption that on real surfaces we can find a neutral plane $h_{0}$, where tension stresses and compression stresses will be equalized. Depending on the type of material, the neutral plane position varies. This plane varies also according to the method of disintegration in various technologies with flexible cutting tools. Whether it is a cold disintegration tool as AWJ (hydroabrasive water jet), or tools with a thermal thermodynamic influence as laser, plasma or oxygen. The common attributes are the total deformation capacity of the surface and the core of material. This is evident from the topographical parameters on newly generated surfaces, 
where is exactly measurable. There is shown a logical zonality in the distribution of topographical elements. Therefore, we can identify similar pattern of deformation at different depth levels, which are reflected in the final surface topography. We have determined a parameter $K_{\text {cut } L}$ $[\mu \mathrm{m}]$ as a new technology-length parameter that increases with the plasticity and deformation capacity of the cut material, and that is determined from three deformation parameters according to

$$
K_{\text {cut } L}=\frac{R a \cdot h}{Y_{\text {ret }}}=\frac{10^{12}}{E_{\text {mat }}{ }^{2}}
$$

A product of roughness and depth divided by trace retardation (at an angle arc $\delta\left[^{\circ}\right]$ ) is constant for all depths and materials. By regression we shall obtain a numerical value of $K_{\text {cut } L}=10^{12} / E_{\text {mat }}^{2}[\mu \mathrm{m}]$, which holds true generally for all materials. A mechanical equilibrium at the depth level of neutral plane $h_{o}$ is defined as (2)

$$
\frac{R a_{0} \cdot h_{0}}{Y_{\text {ret } 0}}-K_{c u t L}=0
$$

From the equation of equilibrium (2) we shall explicitly express and calculate main deformation parameters of the surface, namely $R a, Y_{\text {ret }}, \delta$ in relation to depth $h$, and represent their behaviour in relation to depth of cut $\left(R a, Y_{\text {ret }}\right.$, $\delta)=f(h)$. The main deformation parameters (Fig.5) are expressed by the following relations (3), (4), (5) and (6).

$$
R a=\frac{10^{12} \cdot Y_{r e t}}{V_{d j} \cdot R e^{4} \cdot h},
$$

$$
\begin{gathered}
Y_{r e t}=\frac{10^{-12} \cdot R a \cdot h}{V_{d j} \cdot R e^{4}}, \\
h=\frac{10^{12} \cdot Y_{r e t}}{\left(\operatorname{Re}^{4} \cdot V_{d j} \cdot R a\right)}, \\
\delta=\operatorname{arctg}\left(\frac{Y_{r e t}}{h}\right)
\end{gathered}
$$

In equations (3), (4), (5) a parameter $V_{d j}$ represents a unit volume of deformation expressed by (7)

$$
V_{d j}=R a_{j} \cdot h_{j} \cdot Y_{r e t j},
$$

where $R a_{j}, h_{j}, Y_{\text {retj }}$ are unit parameters. Similarly, equations (8), (9), (10), (11) can be obtained for the main deformation parameters of surface in plane $h_{0}$

$$
\begin{gathered}
R a_{0}=\frac{10^{12} \cdot Y_{r e t 0}}{V_{d j} \cdot R e^{4} \cdot h_{0}}, \\
Y_{r e t 0}=\frac{10^{-12} \cdot R a_{0} \cdot h_{0}}{V_{d j} \cdot R e^{4}}, \\
h_{0}=\frac{10^{12} \cdot Y_{r e t_{0}}}{\left(\operatorname{Re}^{4} \cdot V_{d j} \cdot R a_{0}\right)}, \\
\delta_{0}=\operatorname{arctg}\left(\frac{Y_{r e t 0}}{h_{0}}\right) .
\end{gathered}
$$

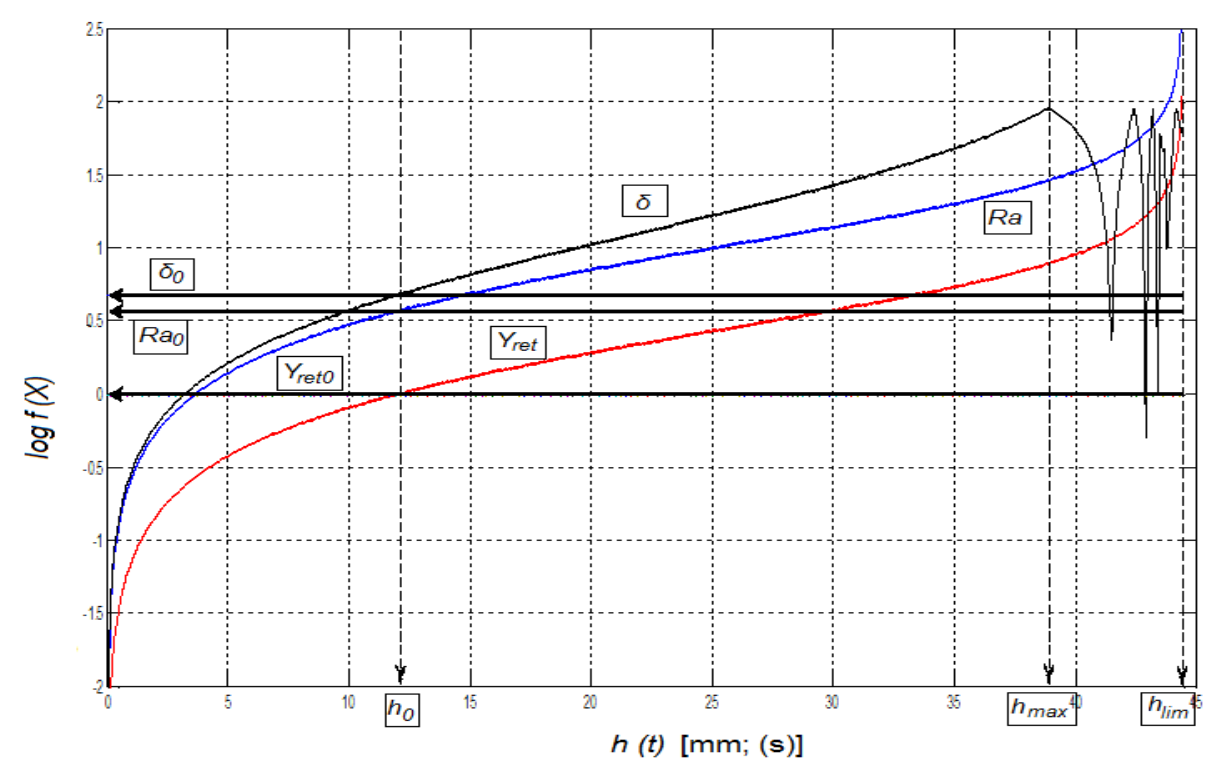

Fig.5. Behaviour of main surface deformation parameters in relation to depth of cut $\left(R a, Y_{\text {ret }}, \delta\right)=\mathrm{f}(h)$ for material steel grade $5 \mathrm{CrNi18}-10$; graphs are plotted in semilog coordinates. 
The graphic dependence $\delta=\mathrm{f}(h)$ shows the limit depth of cut $h_{\text {lim }}$ for a specific material and the decomposition of cut trace after achieving the limit angle of deviation $\delta=90^{\circ}$ within a depth interval $\Delta h=h_{\text {lim (teor) }}-h_{\max }$ (studied hypothetically by authors, e.g. [14], [15], [16], [17], [18]). The theoretical limit depth is determined by an equation (12) in accordance with theory and statistics of results achieved in practice

$$
h_{\text {lim }(\text { teor })}=10^{3} \cdot K_{\text {cut } L}
$$

For the derivation of a basic form of the topographic function for the plane of trace we can define functional relations of stress and deformation quantities to instantaneous cut surface deformation [10]. Thus we shall obtain equations for the decomposition of the concerned quantities to the tension and compression and neutral components, including summarised values. For cumulative stress an equation (13), which contains decomposition components of stress for compression $\sigma_{r z}$ and the decomposition component of stress for tension $\sigma_{r e t}$, is valid:

$$
\left.\sigma_{\text {sum }}=\sqrt{\left(\sigma_{r z}^{2}+\sigma_{r e t}^{2}\right.}\right) .
$$

For particular components, relations (14) to (18) hold true.

$$
\begin{gathered}
\sigma_{r z}=\operatorname{Re} \cdot \sqrt[4]{\frac{K_{p l}}{K_{c u t L}}}, \\
\sigma_{r e t}=\operatorname{Re} \cdot \sqrt[4]{\frac{K_{c u t L}}{K_{p l}}}, \\
E_{\text {sum }}=\sqrt{\left(E_{r z}^{2}+E_{r e t}^{2}\right)}, \\
E_{r e t}=E_{m a t} \cdot \sqrt{\left(\frac{K_{c u t L}}{K_{p l}}\right)} \\
E_{r e t z}=E_{\text {mat }} \cdot \sqrt{\left(\frac{K_{p l}}{K_{c u t L}}\right) .}
\end{gathered}
$$

To the total trace roughness a relation (19) applies.

$$
R a_{\text {sum }}=\sqrt{\left(\frac{1}{R a^{2}}+R a^{2}\right)}
$$

We also derived a more realistic approximation for the calculation of actual surface roughness $R a$ with the use of an auxiliary function which depends on flow stress in the radial direction by equation (20)

$$
R a_{r a d}=R a_{0} \cdot 10^{3} \cdot \frac{\sigma_{r z}}{E_{m a t}}
$$

The main elements of cut wall surface deformation, i.e. surface deformation $K_{p l}=R a \cdot h$ as a function of deformation stress in the cut trace can be quantitatively defined by regression relations. Because also the other main surface deformation parameters are functionally interconnected, it will also generally hold true that $\left(R a_{x}, \delta_{\mathrm{x}}, Y_{\text {retx }}, h_{x}\right)=f\left(\sigma_{r z}\right.$, $\left.\sigma_{\text {sum }}, E_{r z}, E_{\text {sum }}\right)$ in the compression zone, and, reciprocally, also for these quantities in the tension zone. The compression and tension branches intersect at the nodes $\mathrm{A}$, $\mathrm{B}, \mathrm{C}$ in the neutral plane of yield points $h_{0}$ or in the node $\mathrm{E}$ in the plane of elastic limits $h_{e l}$ (Fig.6). Similarity in the behaviour of these functions is evidence of occurrence of deformation owing to a balance between the tension and compression components of stress. The geometric shape of the curve of trend in development of roughness values represents a below derived curve of and equation for the topographic function in the radial direction $R a_{\sigma s u m}=(h)$.

The described working hypothesis and the conclusions enabled us to develop empirically a basic equation for the topographic function $R a_{T b}=f\left(h, \sigma_{r z}, \sigma_{r e t}, \sigma_{\text {sum }}, R e\right)$ in a form (21). The course of this function is shown in Fig.6. The basic equation for topographic function (22) $R a_{T}=f\left(h, \sigma_{r z}\right.$, $\left.\sigma_{\text {ret }}, \sigma_{\text {sum }}, \operatorname{Re}, v_{p}, W_{\text {las }}, p, d, f, z\right)$ is extended to include the influence of technological parameters of laser cutting. The basic equation for the topographic function in the neutral plane is described by the equation (23). The equations are drafted to include the direct influence of technology parameters $v_{p}, W_{\text {las }}, d, p, f, z$ and thus to be able to be used, just as for the determination of optimized values of these process parameters, also for the online control of them.

$$
\begin{gathered}
R a_{T b}=R a_{j} \cdot \mathrm{e}^{\ln \sqrt{(\log h)^{2}+\left(\log \sigma_{\text {sum }}\right)^{2}+\left(\text { Ra } a_{\text {rad }}\right)^{2}}} \\
R a_{T b 0}=R a_{j} \cdot \mathrm{e}^{\ln \sqrt{\left(\log h_{0}\right)^{2}+\left(\log \sigma_{\text {sum } 0}\right)^{2}+\left(\text { Ra }_{\text {rad } 0}\right)^{2}}} \\
\ln \left(\left(e^{\sqrt{f^{2}}}\right) \cdot\left(\frac{p \cdot d \cdot W_{\text {las }}}{v_{p}}\right)^{(0.25 \cdot(f+z))} \cdot\left(R a_{T b}\right)^{0.25^{*}(f+z)}\right)
\end{gathered}
$$

$$
R a_{T 0}=R a_{j} e^{\ln \left(\left(e^{\sqrt{f^{2}}}\right) \cdot\left(\frac{p \cdot d \cdot W_{l a s}}{v_{p}}\right)^{(0.25 \cdot(f+z))} \cdot\left(R a_{T b 0}\right)^{0.25^{*}(f+z)}\right)}
$$

The typical behaviour of the topographic function according to the above-mentioned relation (14) with the subsequent verification of the behaviour according to the values measured by the optical and mechanical profilometers is shown in Fig.7. 


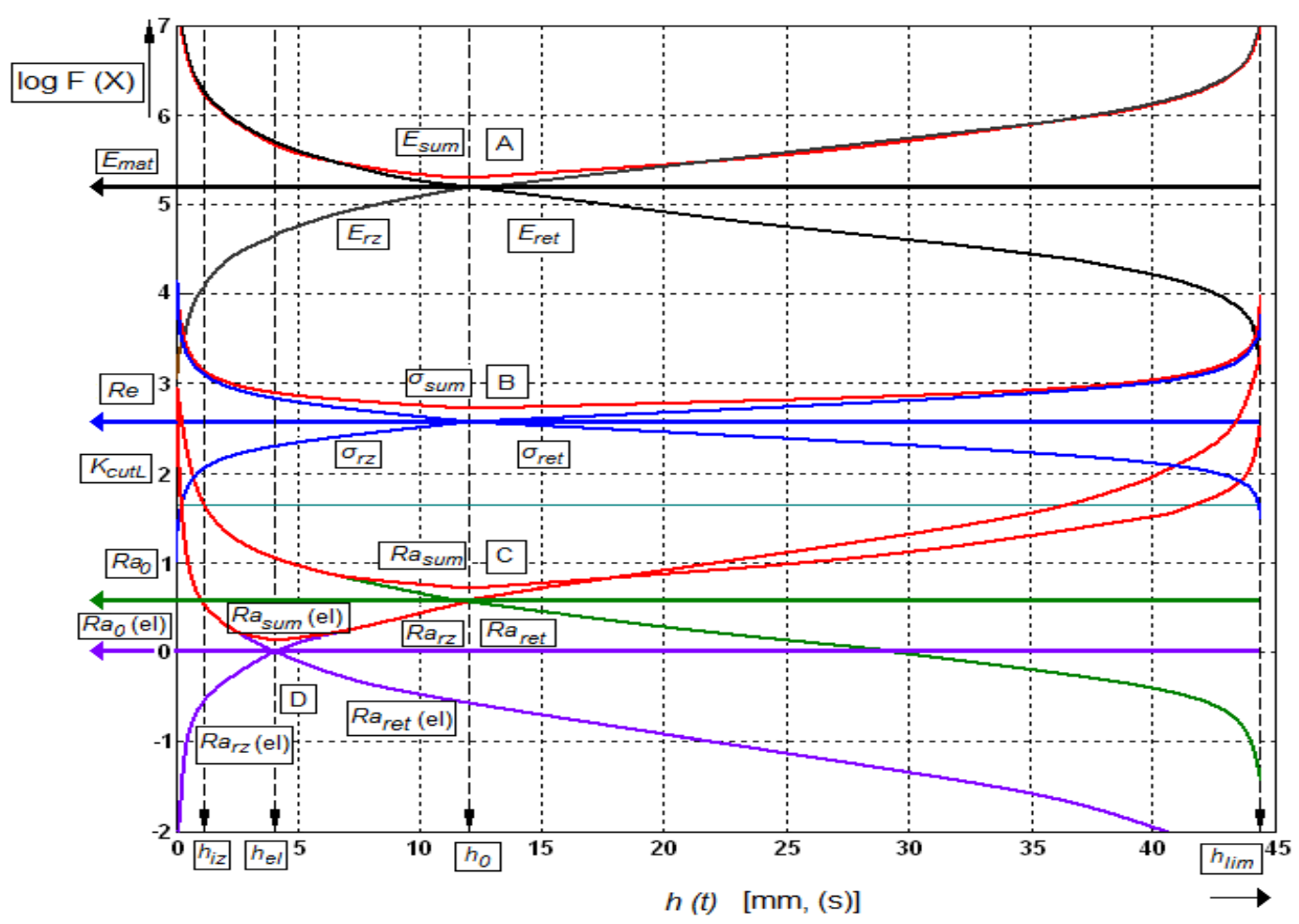

Fig.6. Behaviour of stress deformation components in modular, stress and deformation zones due to external loading for material steel grade $\mathrm{X} 5 \mathrm{CrNi18-10}$; graph is plotted in semilog coordinates.

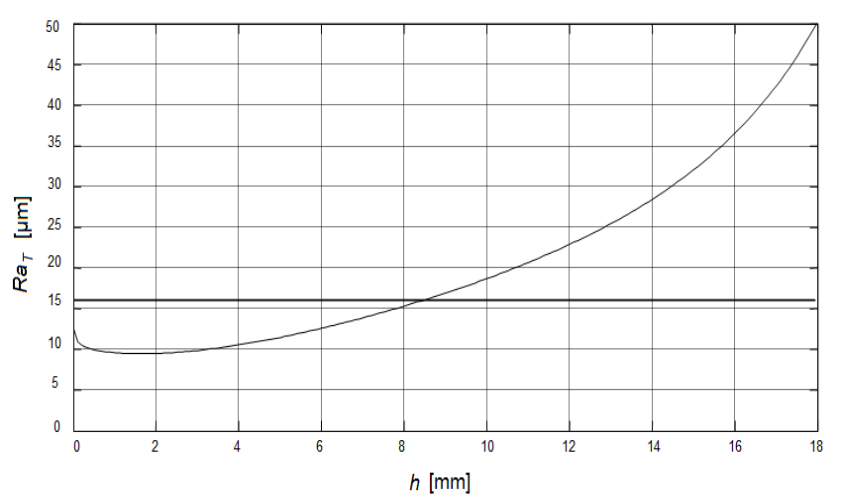

Fig.7. Behaviour of topographic function $R a_{T}$ in relation to depth $h$ for material EN S355J0.

\section{RESULTS AND DISCUSSION}

Results of the proposed topographic function were confronted with [19], where laser cutting process optimization was dealt with. As optimization criterion, minimum surface roughness according to the designed model in the following form (25) there was chosen,

$$
\begin{aligned}
& y_{R a 0}=-0.61 \cdot x_{1}+0.04 \cdot x_{1}^{2}-0.0007 \cdot x_{2}+2.510 \cdot 10^{-6} \cdot x_{2}^{2}-2.979 \cdot x_{3} \ldots \\
& \ldots+0.375 \cdot x_{3}^{2}+4.713 \cdot x_{4}-4.745 \cdot x_{4}{ }^{2}+6.111 \cdot x_{5}-1.072 \cdot x_{5}^{2}-1.397
\end{aligned}
$$

where specific variables $x_{1}, x_{2}$ to $x_{5}$ represent values of the above-mentioned technology parameters represented by graphical dependences, where:
$y_{R a 0}-$ is final surface roughness in the middle line of cut, $x_{1}$ - thickness of the material being cut [mm],

$x_{2}$ - laser cutting speed $\left[\mathrm{mm} \cdot \mathrm{min}^{-1}\right]$,

$x_{3}$ - laser power [kW],

$x_{4}$ - gas pressure [MPa],

$x_{5}$ - nozzle diameter $[\mathrm{mm}]$.

This approach is based on regression analysis in contrast to the stated analytical-empirical approach in the submitted study. It does not contain mechanical parameters of the material, and that is why it cannot be generalized. In Fig. 8 there is a comparison of the results of topographic function $R a_{T}$ according to our proposal and those of topographic function $R a_{H}$ according to [19] at inserting the same technology parameters used really in cutting steel of type EN S355J0 for the theoretical depth $h=60 \mathrm{~mm}$.

By putting $h=h_{0}=15.99 \mathrm{~mm}$ for the given material we shall obtain the value of $R a_{H 0}=26.7 \mathrm{~mm}$ and the value of $R a_{T 0}=29.84 \mathrm{~mm}$ for comparison; the functions intersect at three depth levels and $R a_{T}=R a_{H}$. In Fig.9 to 13, the comparison of values measured using instruments Mitutoyo Surftest SJ401 $\left(R a_{L 1}\right)$ and Talysurf $2001\left(R a_{L 2}\right)$ and the analytical-empirically determined topographic function according to (20) is presented. As can be seen from the presented profiles, there is visible diversity in the roughness distribution for one material obtained at different traverse speeds and at different focal lengths. In general and with physical reasoning, the topographic function is increasing with increasing depth when choosing a positive focal length, and vice versa for negative focal length. 


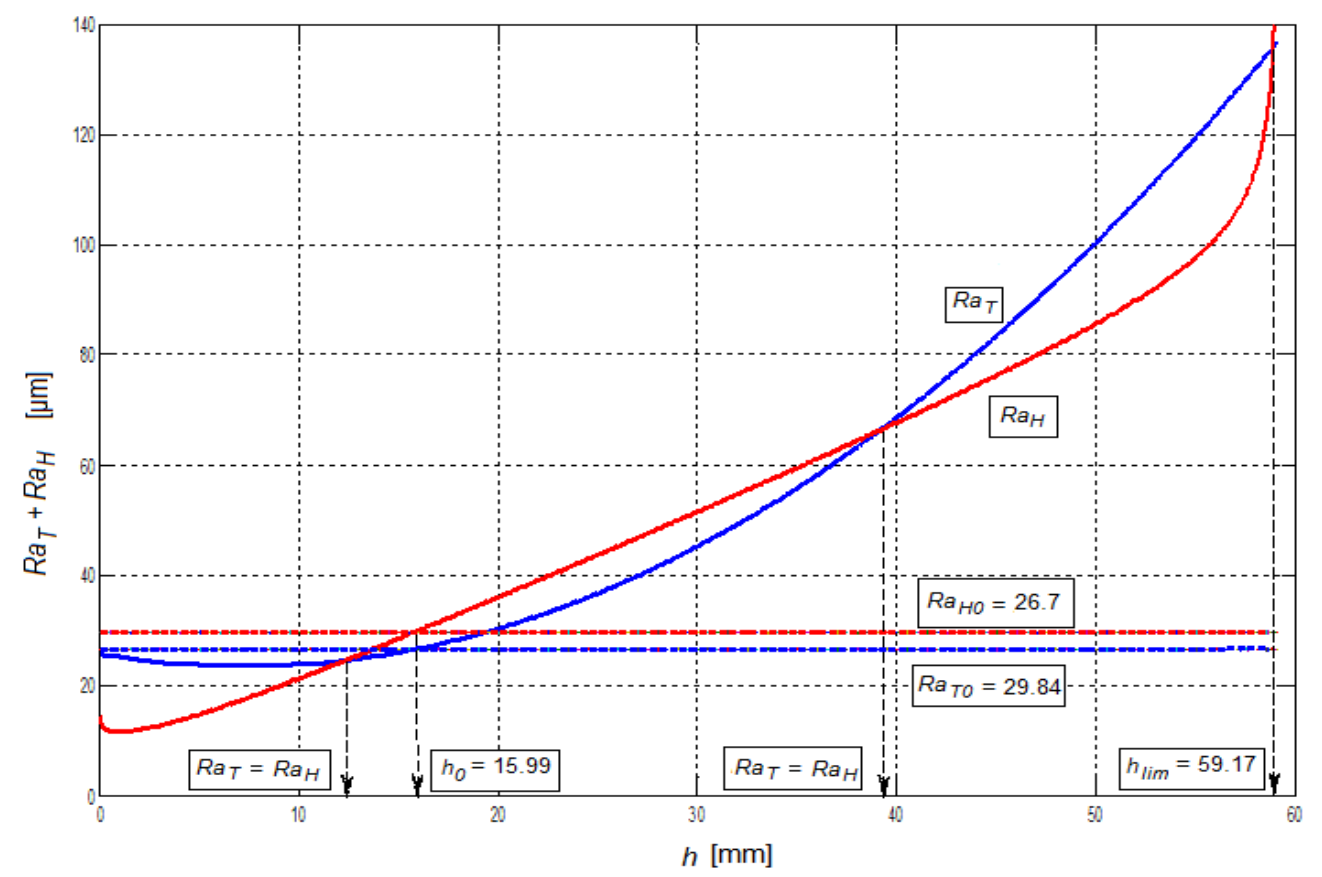

Fig.8. Comparison between the topographic functions $R a_{H}$ and $R a_{T}$.

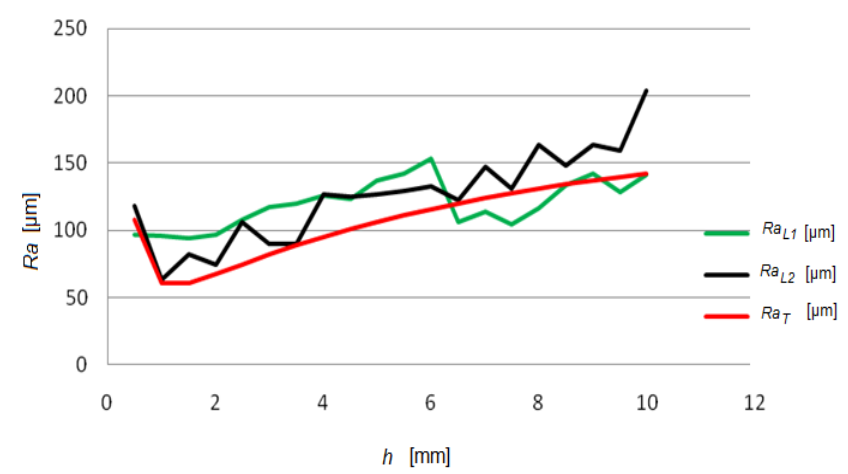

Fig.9. Graphical comparison of topographic function $R a_{T}$ of titanium sample $10 \mathrm{~mm}$ thick at $350 \mathrm{~mm} \cdot \mathrm{min}^{-1}$ speed with $R a_{L I}$ and $R a_{L 2}$ data, with focal length $f$ of +3.5 .

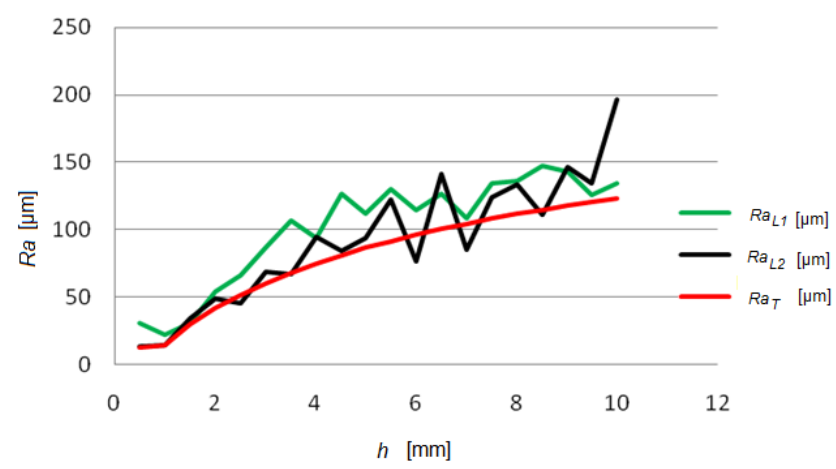

Fig.10. Graphical comparison of topographic function $R a_{T}$ of titanium sample $10 \mathrm{~mm}$ thick at $450 \mathrm{~mm} \cdot \mathrm{min}^{-1}$ speed with $R a_{L I}$ and $R a_{L 2}$ data, with focal length $f$ of +3 .

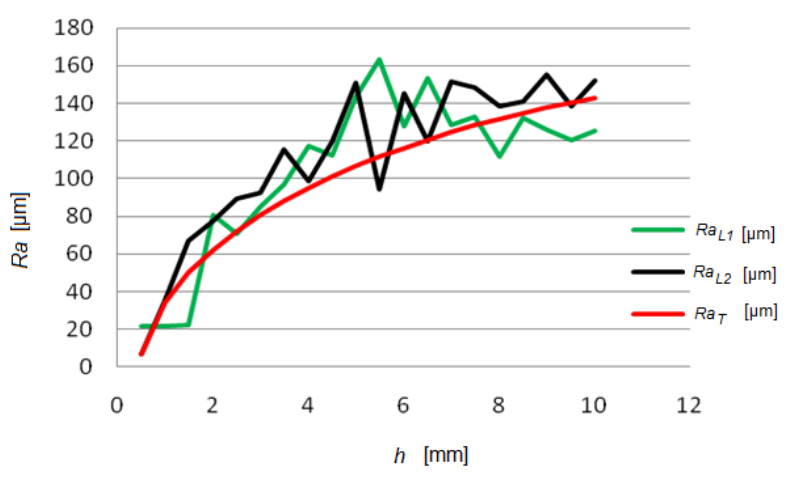

Fig.11. Graphical comparison of topographic function $R a_{T}$ of titanium sample $10 \mathrm{~mm}$ thick at $550 \mathrm{~mm} \cdot \mathrm{min}^{-1}$ speed with $R a_{L l}$ and $R a_{L 2}$ data, with focal length $f$ of +2 .

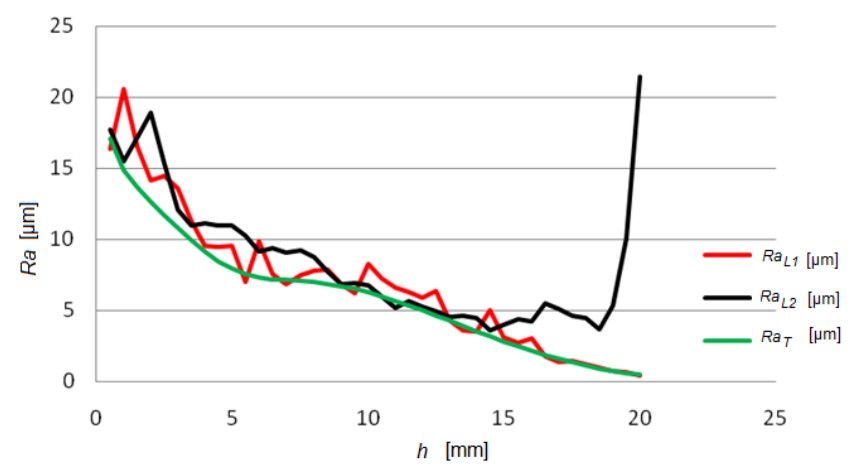

Fig.12. Graphical comparison of topographic function $R a_{T}$ of sample (EN S355J0) $20 \mathrm{~mm}$ thick with $R a_{L 1}$ and $R a_{L 2}$ data, with focal length $f$ of -3 . 


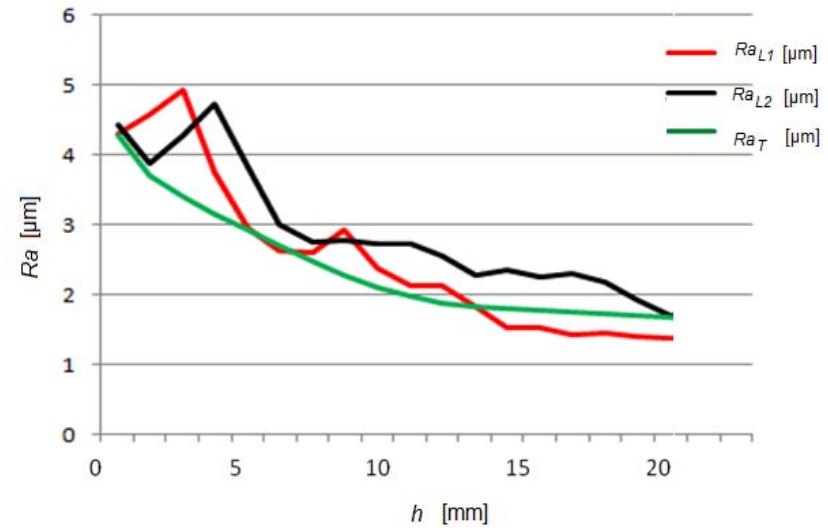

Fig.13. Graphical comparison of topographic function $R a_{T}$ of sample (X5CrNi18-10) $20 \mathrm{~mm}$ thick with $R a_{L 1}$ and $R a_{L 2}$ data, with focal length $f$ of -2 .

In the course of derivation, the inclusion of main technology variables into the equations had to be respected. By modification of basic forms of equations for $R a_{T}$ and $R a_{T 0}$ we then can calculate functional relations between topography and technology, and thanks to these analyses we can optimize and control the process. From equation (22), we obtain a dimensionless technological constant $K_{\text {tech }}(26)$, if $K_{\text {tech }}$ equals to 1 , we obtain the optimal technological parameters. If $K_{\text {tech }}$ is not equal to 1 , the technological parameters are not optimized. In the calculation of optimized technology parameters, the equation for $R a_{T 0}$ (23) in the neutral plane $h_{0}$ is used as a basis. Calculation and determination of each paramater shall be solved by elimination of selected parameter from Eq.(23). At the neutral plane $h_{0}$, we can obtain (e.g for titanium) the optimized values for gas pressure $p_{\text {opt }}$ of $3.15 \mathrm{MPa}$, nozzle diameter $d_{\text {opt }}$ of $1.85 \mathrm{~mm}$, laser power $W_{\text {lasopt }}$ of $1822 \mathrm{~W}$ and traverse speed $v_{\text {pLopt }}$ of $1990 \mathrm{~mm} \cdot \mathrm{min}^{-1}$. Similarly, the optimized values are obtained for focus lenght $f_{\text {opt }}$ of $0.6 \mathrm{~mm}$ and standoff distace $z_{\text {opt }}$ of $0.7 \mathrm{~mm}$.

$$
K_{\text {tech }}=\exp \left(\left(f^{2}\right)^{0.5}\right) \cdot\left(\frac{p \cdot d \cdot W_{\text {las }}}{v_{p}}\right)^{(0.25 \cdot(f+z))}
$$

Then, the optimized technology constant is given by (27)

$$
K_{\text {techopt }}=\exp \left(\left(f_{\text {opt }}^{2}\right)^{0.5}\right) \cdot\left(\frac{p_{\text {opt }} \cdot d_{\text {opt }} \cdot W_{\text {lasopt }}}{v_{\text {popt }}}\right)^{\left(0.25 \cdot\left(f_{\text {opt }}+z_{\text {opt }}\right)\right)}
$$

and an indicative ratio needed for an engineering technologist is given by (28)

$$
I N D_{\text {tech }}=\frac{K_{\text {tech }}}{K_{\text {techopt }}} .
$$

If we compare the calculated values with individually selected parameters in Table 1 and put these values into the given equations, we shall obtain $K_{\text {techopt }}$ of 3.21 and $K_{\text {tech }}$ from 3.44 to 4.32 for different speeds. Hence, the increase in surface layer load in the cut (up 34.6\%) is associated with re-melting the material and decreasing the limit depth of cut. There is a significant difference in performance, productivity and economy of operation. Based on the optimized speed compared to individually chosen one, the difference in performance and economic parameters is almost four-time higher. Distribution of roughness $R a_{T}$ in Fig. 14 is calculated by substituting the optimized parameters into the equation (22) and by changing the focus length from $f=-4.5$ to $0 f=+3.5$ at a constant standoff distance $z$ of 1 $\mathrm{mm}$. Theoretically, the smoothest surface has been reached at the given standoff distance $z$ and focal length $f$ of -1 .

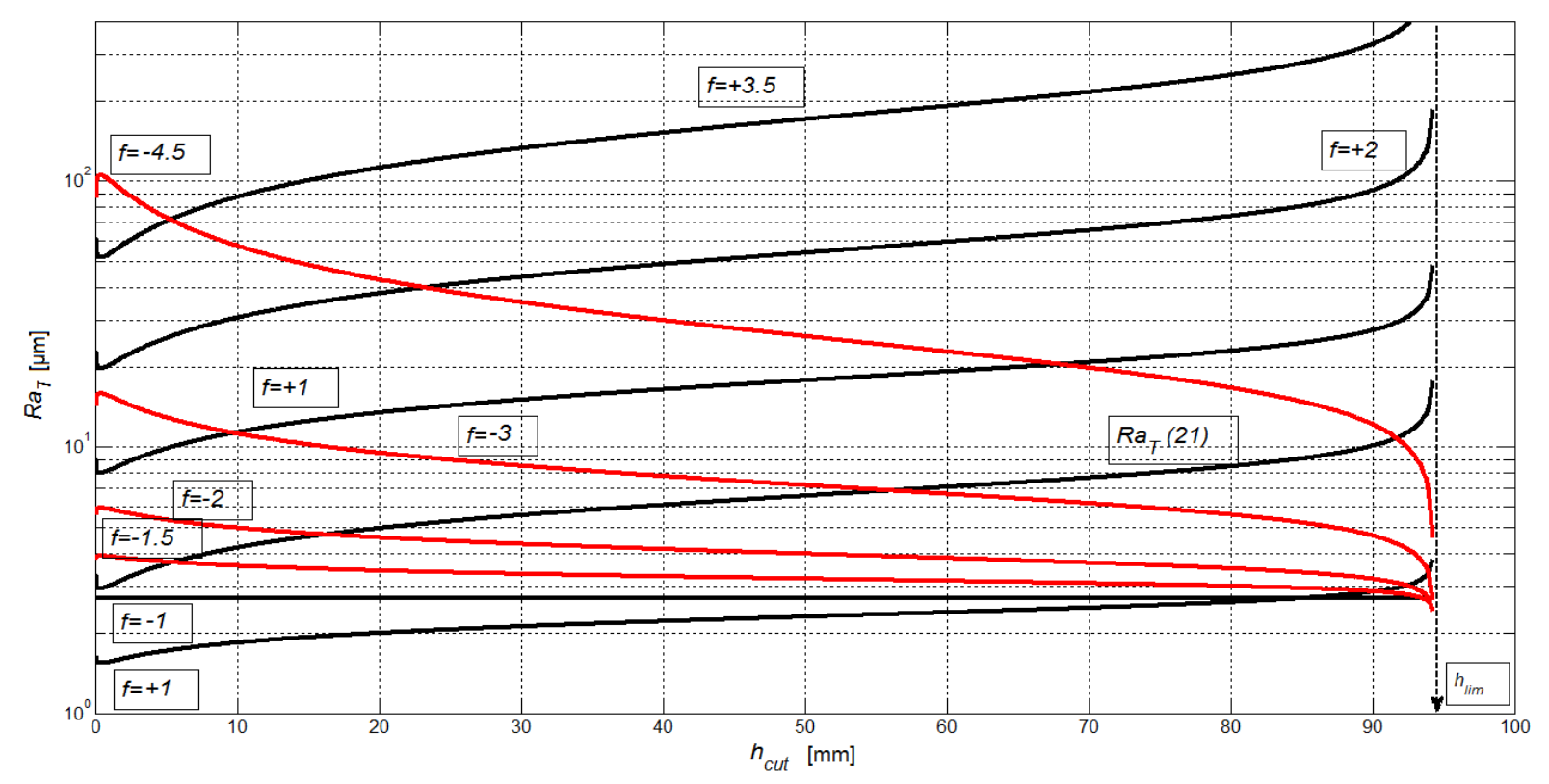

Fig.14. Distribution of roughness $R a_{T}$ for titanium to the limit depth of cut. 


\section{CONCLUSION}

The aim of the contribution was a proposal for assessing the quality of cut in the case of laser beam cutting technology. The analysis of the problem, verification of concept of solving, selection of experimental procedures and choice of experimental methods, measurements themselves were carried out. On the basis of analysis and interpretation of acquired surface data, a topographic function, which is a basis for the prediction of quality and control of the laser cutting technology was derived in an original way. In the framework of interpretation of measured values, relations between geometric and technology parameters are systematically analysed and physico-mechanical and distribution principles followed by them are formulated. The comprehensive topographic function for roughness in the radial direction is determined according to (22). The equations are drafted to include the direct influence of technology parameters $v_{p}, W_{\text {las }}, d, p, f, z$ and thus to be able to be used, just as for the determination of optimized values of these process parameters, also for the online control of them. Physical equations are in general applicable for all materials. The proposed method of calculation is treated as a separate algorithm for practical use. The whole calculation can be performed as functions in MATLAB and EXCEL. It also presents a possibility to predict real measured values of roughness in materials that exceed cutting depth of the used samples; it is done for a further theoretical development at this area. The graphs come out from the derived physical equations and it is needed to solve them separately for every single material. The basic derived physical equations apply to all technical materials in general. In this paper, we present a practical example of control of technological parameters for titanium. Furthermore, the solution enables the prediction of process functions of technological process for any material including technological parameters and an interactive modelling of technological process.

\section{ACKNOWLEDGMENT}

This paper has been elaborated in the framework of the IT4Innovations Centre of Excellence project, reg. no. CZ.1.05/1.1.00/02.0070 supported by Operational Programme 'Research and Development for Innovations' funded by Structural Funds of the European Union and state budget of the Czech Republic, the RMTVC project No.CZ.1.05/2.1.00/01.0040, and the project Integrita No.CZ.1.07/2.3.00/20.0037.

\section{REFERENCES}

[1] Yilbaş, B.S. (1996). Experimental investigation into $\mathrm{CO}_{2}$ laser cutting parameters. Journal of Materials Processing Technology, 58 (2-3), 323-330.

[2] Riveiro, A., et al. (2010). Parametric investigation of $\mathrm{CO}_{2}$ laser cutting of 2024-T3 alloy. Journal of Material Processing Technology, 210 (9), 1138-1152.

[3] Scintilla, L.D., Tricarico, L. (2012). Estimating cutting front temperature difference in disk and $\mathrm{CO}_{2}$ laser beam fusion cutting. Optics \& Laser Technology, 44 (5), 1468-1479.
[4] Eltawahni, H.A., Hagino, M., Benyounis, K.Y., Inoue, T., Olabi, A.G. (2012). Effect of $\mathrm{CO}_{2}$ laser cutting process parameters on edge quality and operating cost of AISI316L. Optics and Laser Technology, 44 (4), 1068-1082.

[5] Sharma, A., Yadava V. (2012). Modelling and optimization of cut quality during pulsed Nd:YAG laser cutting of thin Al-alloy sheet for straight profile. Optics \& Laser Technology, 44 (1), 159-168.

[6] Cekic, A., Kulenovic, M., Begic, Dj. (2008). Roughness as parameter of cut quality during $\mathrm{CO}_{2}$ laser cutting of high alloy steels for the special purpose. In Proceedings of the 19th International DAAAM Symposium, 22-25 October 2008. Wienna, Austria: DAAAM International, 225-226.

[7] Jena, D.P., Kumar, R. (2011). Implementation of wavelet denoising and image morphology on welding image for estimating HAZ and welding defects. Measurement Science Review, 11 (4), 108-111.

[8] Ghany, K.A., Newishy, M. (2005). Cutting of $1.2 \mathrm{~mm}$ thick austenitic stainless steel sheet using pulsed and CW Nd: YAG laser. Journal of Material Processing Technology, 168 (3), 438-447.

[9] Di Pietro, P., Yao, Y.L. (1994). An investigation into characterizing and optimizing laser cutting quality. International Journal of Machine Tools and Manufacture, 34 (2), 225-243.

[10] Valíček, J., Harničárová, M., Čep, R., Rokosz, K., Łukianowicz, C., Kozak, D., Zeleňák, M., Koštial, P. (2012). Surface quality control of materials being cut by laser with respect to corrosion resistance. Defect and Diffusion Forum, 326-328, 324-329.

[11] Neslusan, M., Mrkvica, I., Čep, R., Kozak, D., Konderla, R. (2011). Deformations after heat treatment and their influence on cutting process. Tehnički Vjesnik (Technical Gazette), 18 (4), 601-608.

[12] Łukianowicz, Cz., Karpiński, T. (2001). Optical system for measurement of surface form and roughness. Measurement Science Review, 1 (1), 151-154.

[13] Domotor, Z., Batitsky, V. (2009). The analytic versus representational theory of measurement: A philosophy of science perspective. Measurement Science Review, 8 (6), 129-146.

[14] Hashish, M. (1984). A model study of metal of cutting with abrasive water jet. Journal of Engineering Materials and Technology, 106 (1), 88-100.

[15] Hashish, M. (1992). A modeling study of jet cutting surface finish. PED, 58, 151-167.

[16] Hashish, M. (1988). Visualization of the abrasivewaterjet cutting process. Experimental Mechanics, 28 (2), 159-169.

[17] Hassan, I.A., Chen, C., Kovacevic, R. (2004). Online monitoring of depth of cut in AWJ cutting. International Journal of Machine Tools \& Manufacture, 44 (6), 595-605. 
[18] Kovacevic, R., Mohan, R., Zhang, Y.M. (1995). Cutting force dynamics as a tool for surface profile monitoring. Journal of Engineering for Industry, 117 (3), 340-350.
[19] Hrabčáková, I. (2003). Simulation and optimization of a quality and reliability of automated control of production processes in engineering applications in the technology of laser cutting. Dissertation Thesis. Prešov.

Received June 18, 2012. Accepted September 14, 2012. 